\title{
LASER EXCITATION SPECTRA AND LIFETIME MEASUREMENTS OF THE CO $d\left({ }^{3} \Delta_{i}\right)$ STATE
}

\author{
BRADLEY E. MILLER $\dagger$ and PETER A. SCHULZ \\ Georgia Institute of Technology, Atlanta, Georgia
}

(Received 25 March 1991; in final form 8 May 1991)

CO in a supersonic pulsed beam is excited to the $d^{3} \Delta_{i}$ state by VUV photons generated in a nonlinear four-wave mixing scheme. An excitation spectrum of the $\operatorname{CO} d^{3} \Delta_{i}\left(v^{\prime}=20, J^{\prime}=1-3\right)$ is obtained at a resolution of $0.8 \mathrm{~cm}^{-1}$ for the first time. The radiative lifetimes of the rotational states are measured as $1.70 \pm 0.08 \mu \mathrm{s}$, which are similar to but shorter than the lifetimes associated with lower vibrational levels.

KEY WORDS: Laser excitation spectra, radiative lifetimes, carbon monoxide.

\section{BACKGROUND}

Carbon monoxide has been extensively studied in the infrared region ${ }^{1-4}$ of the spectrum yielding a wealth of information on its ground state. Absorption and fluorescence spectra at ultraviolet wavelengths have identified several excited electronic states. ${ }^{5-10}$ For the most part, the upper singlet states have been well characterized because the transition to and from the $\operatorname{CO} X^{1} \Sigma^{+}$ground state is spin allowed. However, characterizing triplet states whose transitions with the $\operatorname{CO} X^{1} \Sigma^{+}$ ground state are spin-forbidden is difficult. Often times these states can only be probed if there exists a strong perturbation between the singlet state and the triplet state in question. Non-optical excitation such as electron bombardment is one way to circumvent the complications inherent to a spin-forbidden process, although the nonselectivity of this process poses other problems. These forbidden transitions may also be investigated using a high intensity photon source to overcome the low transition probability. This strategy is pursued with lasers and nonlinear optics in this study of the $d^{3} \Delta_{i}$ state of CO.

The $d^{3} \Delta_{i}$ state of CO has previously been observed in two electronic transitions: (a) the $d^{3} \Delta_{i}-a^{3} \pi$ emission bands, often referred to as the triplet bands, ${ }^{11}$ and (b) the $d^{3} \Delta_{i}-X^{1} \Sigma^{+}$absorption bands. It was noted by Birge ${ }^{12}$ that the lower electronic state of this transition is identical with the lower state of the third positive bands $\left(b^{3} \Sigma^{+}-a^{3} \pi\right)$, implying that the upper state is triplet in nature. Birge's original vibrational assignments were revised by Asundi $^{13}$ and once again by Herzberg. ${ }^{14}$

After analyzing the rotational structure of five triplet bands, Gero and Szabo ${ }^{15}$ concluded that the transition of $d^{3} \pi-a^{3} \pi$. In 1958 Mulliken $^{16}$ predicted that an

† Current address: Center for Naval Analyses, Alexandria, VA 22303-0268.

¥ Current address: MIT Lincoln Laboratory, Lexington, MA. 
electronic state of ${ }^{3} \Delta$ symmetry rather than ${ }^{3} \pi$ should lie near the observed energy of the $d$ state. Lefebvre-Brion, Moser and Nesbet ${ }^{17}$ reached the same conclusion after carrying out more detailed calculations. The point was settled in 1962 after Carroll ${ }^{18}$ performed detailed rotational analyses and concluded that the upper state was indeed $d^{3} \Delta$. Herzberg and co-workers obtained precise values for the vibrational and rotational constants by studying the $\operatorname{CO} d^{3} \Delta_{i}-X^{1} \Sigma^{+}$absorption spectrum up to $v^{\prime}=22$ in the $d$ state.

Much of the previous work on the $\mathrm{CO}$ molecule depended on the perturbation between the $A^{1} \pi$ and $d^{3} \Delta_{i}$ states. ${ }^{6,14,18-21}$ This coupling of states manifests itself in measurements of the $d$ state lifetime. Perturbed states are more correctly described as an admixture of the pure states:

$$
\Psi=C_{1} \Psi_{A}+C_{2} \Psi_{d}
$$

where $\Psi_{A}$ and $\Psi_{d}$ are the non-interacting $A$ and $d$ state wavefunctions of $C_{1}$ and $C_{2}$ are numerical coefficients with $C_{1}^{2}+C_{2}^{2}=1$. The unperturbed lifetimes for the $A-X$ and $d-a$ transitions are on the order of $\tau_{1}=10 \mathrm{~ns}$ and $\tau_{2}=6 \mu$ s respectively. ${ }^{21}$ If the lifetime of a highly perturbed $d^{3} \Delta_{i}$ state is measured it will be seen to be considerably shorter than those of unperturbed states because of the influence of the short lifetime $A^{1} \pi$ state. The lifetime of such a state is given by the following relation:

$$
\frac{1}{\tau}=\frac{C_{1}^{2}}{\tau_{1}}+\frac{C_{2}^{2}}{\tau_{2}}
$$

with $\tau_{1}$ and $\tau_{2}$ the unperturbed lifetimes mentioned above.

Lifetimes for the triplet system were first reported by Fowler and Holzberlein. ${ }^{19}$ They were only able to measure a fast $31 \mathrm{~ns}$, decay and did not resolve the vibrational levels. This lifetime was probably due to a $B^{1} \Sigma^{+}-A^{1} \pi$ transition instead of the desired $d^{3} \Delta_{i}-a^{3} \pi$ transition. Vibrationally resolved lifetimes on the order of $5 \mu \mathrm{s}$ were later obtained by Wentink et al. ${ }^{20}$ for the $d^{3} \Delta_{i}, v^{\prime}=6-10$, levels. Slanger and Black ${ }^{21}$ reported radiative lifetimes for the $d^{3} \Delta_{i}\left(v^{\prime}=5\right)$ level strongly depend on the rotational quantum number, $J$, for $\Omega=1$, which is expected for highly perturbed states. More recent studies by Paske and co-workers ${ }^{22}$ using a delayed coincidence technique tend to refute this finding, although they agree that the mixing with the $A^{1} \pi$ does account for the lifetime. Paske et al. results show that the $J$ dependence seen by Slanger and Black is possibly due to spectral overlap with the $B^{1} \pi\left(v^{\prime}=0\right)-A^{1} \pi\left(v^{\prime}=3\right)$ transition.

In recent years the $\mathrm{CO}$ molecule has been the object of several investigations involving the methods of laser spectroscopy. Since the excited electronic states of $\mathrm{CO}$ lie at least $50,000 \mathrm{~cm}^{-1}$ above the thermally populated levels of the ground state, the one-photon absorption transitions are located almost entirely in the vacuum ultraviolet (VUV) spectral region. The first investigations using lasers employed the method of multiphoton excitation. ${ }^{23,24}$ Single photon excitation spectroscopy also became feasible with the advent of nonlinear optics for the production of narrowband and tunable vacuum ultraviolet radiation by four-wave frequency mixing. The high intensities $\left(10^{11}\right.$ photons/s), high resolution (up to $\left.0.1 \mathrm{~cm}^{-1}\right)$ and short pulse durations also make this an ideal source for performing fluoresence lifetime studies. 


\section{EXPERIMENTAL ARRANGEMENTS}

The two technologies of four-wave sum frequency mixing and supersonic molecular beams were combined to perform high resolution spectroscopy in the vacuum ultraviolet (near $1250 \AA$ ). The general design of the experiment is shown in Figure 1 . VUV radiation is generated in a manner similar to Tomkins and Mahon. ${ }^{25}$ Two Littman-type dye lasers are pumped by the second harmonic of a Nd:YAG laser (Quanta Ray DCR-2) pulsing at $10 \mathrm{~Hz}$. One dye laser is tuned to $6257 \pm 0.2 \AA$, twice amplified to $15 \mathrm{~mJ} /$ pulse, then frequency doubled in a KDP crystal in order to be two photon resonant with the $6 s^{2}-6 s 7 s$ mercury transition. The second dye laser is tunable from $6100 \AA$ to $6350 \AA$ and is amplified once, then combined with the UV beam by means of a dichroic mirror. The now colinear light beams are focused into the center of a mercury cell by a $25 \mathrm{~cm}$ focal length quartz lens. Mercury, the non-linear medium for the four wave mixing scheme, is heated to $180^{\circ} \mathrm{C}$ where it achieves a vapor pressure of about 10 torr. ${ }^{26}$ Approximately 5 torr of helium is used as a buffer gas for anomalous dispersion. The VUV light generated in this process exits the mercury cell through a $\mathrm{MgF}_{2}$ window. A VUV filter (Acton Research) transmits $20 \%$ of the $1250 \AA$ light and rejects most other wavelengths. A typical photon fiux in the reaction chamber is $4 \times 10^{10}$ photons/sec. Finally, this radiation passes through the reaction cell and is detected in an acetone ionization chamber. The acetone is maintained near 500 mtorr and is separated from the experimental chamber by a $\mathrm{MgF}_{2}$ window. A picoammeter detects the ionization current and this signal is then modified by a voltage to frequency converter for collection by a pulse counter.

The carbon monoxide is admitted into the reaction chamber through a $0.5 \mathrm{~mm}$ diameter pulsed valve (Lasertechnics LPV and Model 203 Valve Driver) synchronized with the YAG laser. The vacuum chamber is pumped down to $10^{-6}$ torr when the pulsed valve is closed and a pressure of $5 \times 10^{-5}$ torr is maintained during normal operations. Carbon monoxide gas obtained from Matheson is used without further purification and is held at room temperature in the stagnation chamber at a typical pressure of 1000 torr. The $\mathrm{CO}$ molecules in the pulsed supersonic beam interact with the VUV light within the vacuum chamber. Fluorescence from the reaction is detected by a UV sensitive PMT situated perpendicular to the plane of the beams. The PMT response is monitored as a function of the VUV frequency in order to obtain an excitation spectrum. A gate/delay generator is used to delay detection $100 \mathrm{~ns}$ after each laser pulse. The gate width is $4.0 \mu \mathrm{s}$. Lifetime data are gathered by changing the PMT delay relative to the laser pulse and monitoring the fluorescence in a $0.3 \mu$ s box. All fluorescence intensities are normalized by the VUV intensity.

\section{EXPERIMENTAL OBSERVATIONS}

\section{$\operatorname{CO} d^{3} \Delta_{\mathrm{i}}-\mathrm{X}^{1} \Sigma^{+}$Excitation Spectra}

Interpretation of Herzberg's ${ }^{14}$ results from high vibrational levels $\left(v^{\prime}=19\right.$ and $\left.v^{\prime}=22\right)$ led to a predicted frequency of $80,026 \mathrm{~cm}^{-1}$ for the CO $d^{3} \Delta_{i}\left(v^{\prime}=20\right.$, $J^{\prime}=0$ ) state. Therefore, we began our search for triplet state fluorescence near this 


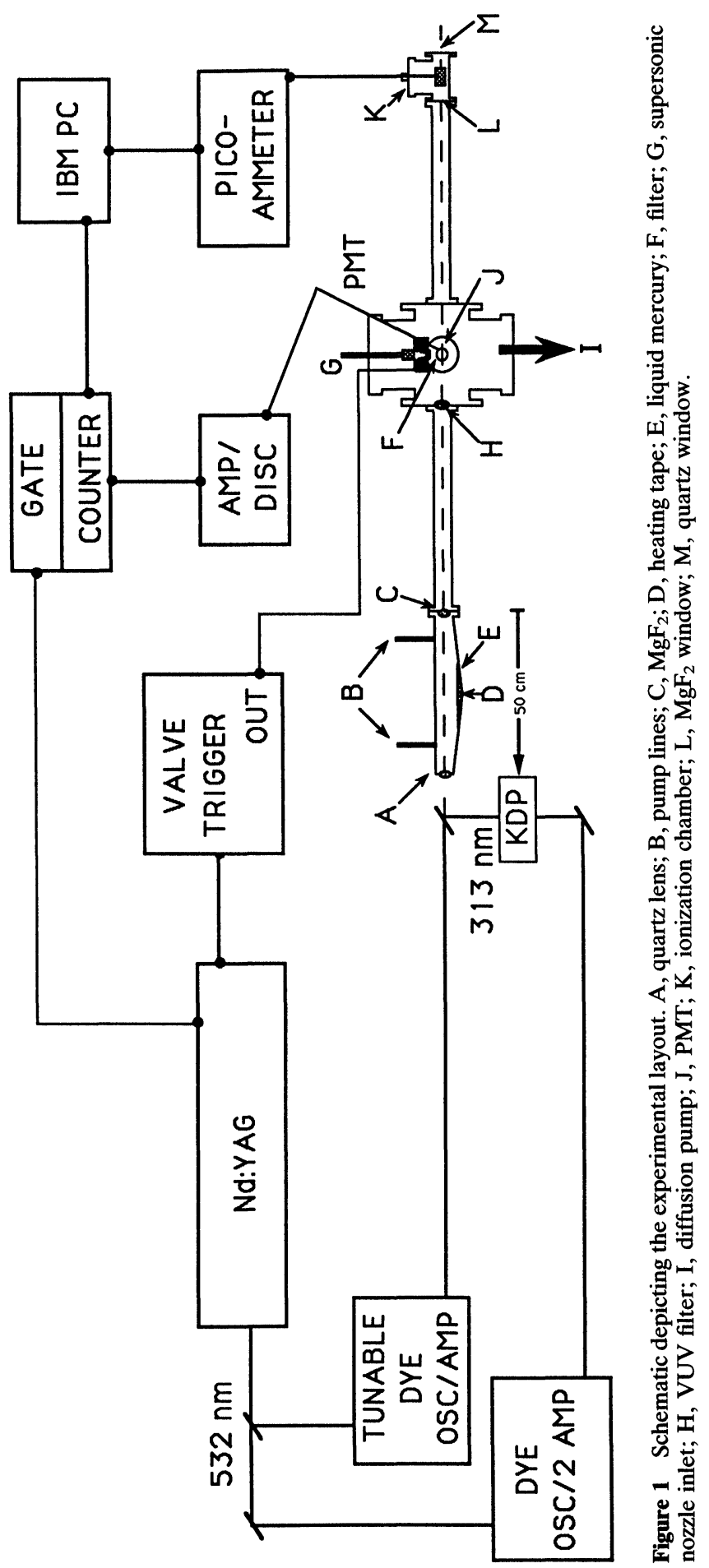




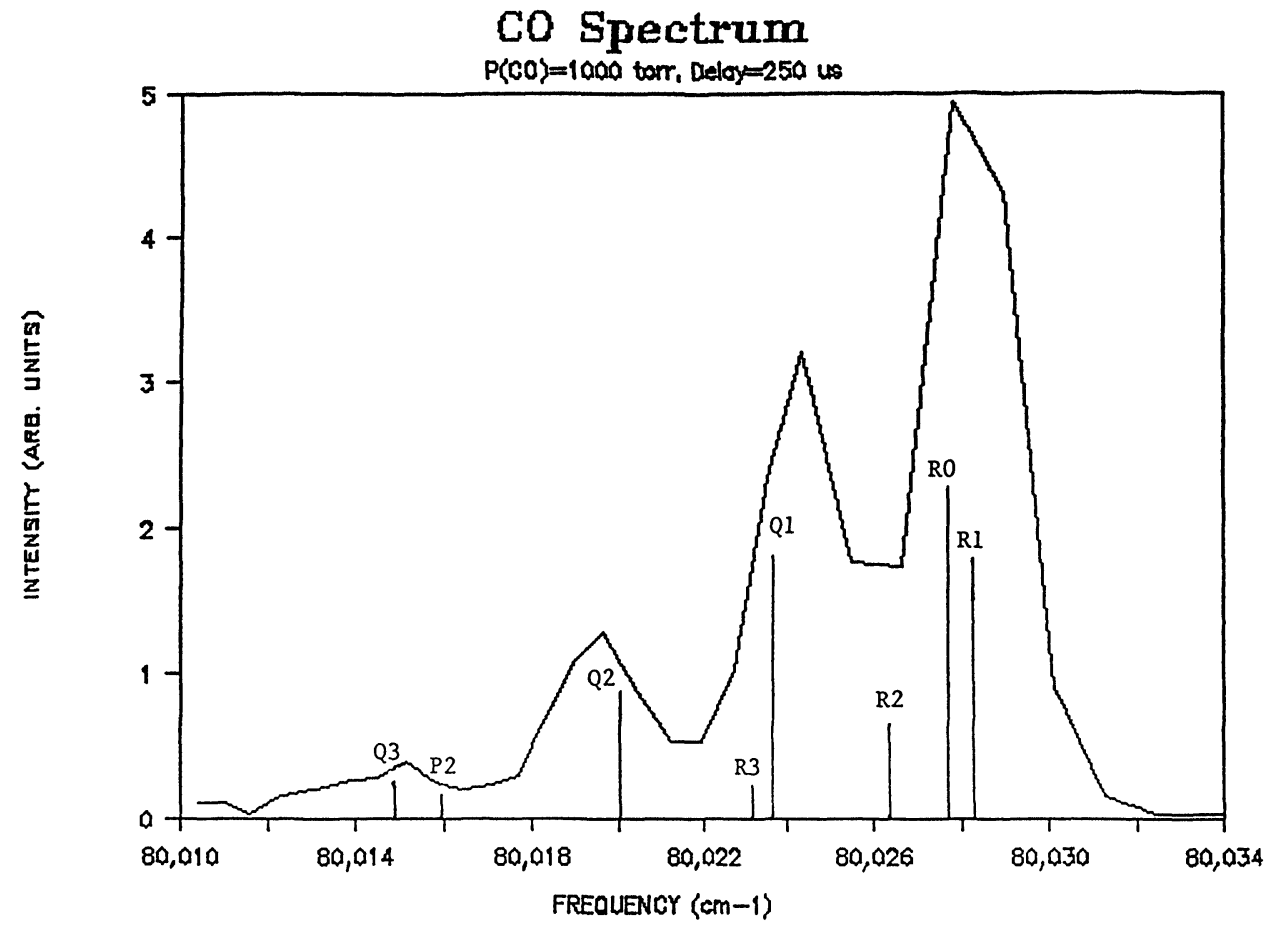

Figure $2 \mathrm{CO}$ excitation spectrum of the primary molecular beam pulse. Rotational assignments are indicated as well as theoretical intensities. A rotational temperature of $9.1 \pm 1.9$ Kelvin is calculated.

excitation energy. The dye laser was scanned in frequency from $80,005 \mathrm{~cm}^{-1}$ to $80,035 \mathrm{~cm}^{-1}$. Figure 2 shows the results. This narrow bandwidth scan, with a resolution of $0.8 \mathrm{~cm}^{-1}$, clearly shows an intense peak at an excitation energy near $80,028 \mathrm{~cm}^{-1}$ and other features indicative of rotational structure.

We made rotational assignments for the observed spectrum with the aid of the rotational constants contained in Tilford and Simmon's reference data ${ }^{27}$ (Table 1). That only four distinguishable peaks are observed is evidence that rotationally cold molecules are created in the supersonic expansion beam. In general the allowed rotational transitions are $\Delta J=-1,0,1$, which correspond to the $\mathrm{P}, \mathrm{Q}$ and $\mathrm{R}$

Table 1 Vibrational and rotational constants of $\mathrm{CO}$

\begin{tabular}{lcc}
\hline & $d^{3} \Delta_{i}$ & $X^{1} \Sigma^{+}$ \\
\hline$T_{e}$ & $61,120.1 \mathrm{~cm}^{-1}$ & $0.0 \mathrm{~cm}^{-1}$ \\
$\omega_{e}$ & $1,171.94$ & $2,169.81$ \\
$\omega_{e} x_{e}$ & 10.635 & 13.288 \\
$\omega_{e} y_{e}$ & 0.0785 & 0.0105 \\
$B_{e}$ & 1.3108 & 1.931 \\
$\alpha_{e}$ & 0.01782 & 0.0175 \\
$D_{e}$ & $6.12 \times 10^{-6}$ & $6.59 \times 10^{-6}$ \\
\hline
\end{tabular}


branches respectively. If the $\mathrm{CO} X^{1} \Sigma$ ground state is sufficiently cooled so that only a few rotational states are formed, then only a few transitions to the $d^{3} \Delta_{i}$ excited state will be seen in accordance with the selection rule above.

The $d^{3} \Delta_{i}$ excited state degeneracies are characterized by the total electronic angular momentum, $\Omega$. For slow rotations Hund's case (a) applies, and the electronic orbital angular momentum and the nuclear angular momentum, $N$, are weakly coupled such that $J=\Omega+N$. Because the selection rule of $\Omega$, when $\Omega$ is a good quantum number, states that $\Delta \Omega=-1,0,1$, only the ${ }^{3} \Delta_{1}-{ }^{1} \Sigma^{+}$sub-band is an allowed transition. Since $\Omega=1$ is the lowest value for a ${ }^{3} \Delta$ state, $J$ cannot be less than unity. As $J$ increases, the rules for Hund's case (a) are no longer strictly valid since $\Omega$ is no longer well defined. In this regime Hund's case (b) applies and transitions to the $d^{3} \Delta_{\Omega}=2,3$ states are also allowed.

The peaks, as labeled in Figure 2, illustrate that only the rotational levels up to $J=2$ are appreciably populated in the ground state. The rotational temperature can be determined by comparing the relative intensities of the different $J$ levels and assuming a Boltzmann distribution with line strengths determined by Hönl-London coefficients. ${ }^{28}$ The predicted intensities are indicated by the line heights for the labeled transitions. As is expected the $Q$ and $R$ branches are much stronger than the $P$ branch.

Fitting the spectrum in Figure 2 to such a distribution, the rotational temperature is found to be $T_{\text {rot }}=9.1 \pm 1.9 \mathrm{~K}$. The measured rotational temperatures are approximately $2 \mathrm{~K}$ greater than the translational temperatures ${ }^{29}$ predicted for the supersonic molecular beam expansion. At such low temperatures we should only see peaks in our spectra up to the $J^{\prime \prime}=2$ line. Klopotek and Vidal ${ }^{30}$ obtained room temperature spectra on lower vibrational states using a similar excitation process. Their spectra show the rotational transitions peak near $J^{\prime \prime}=7$. Klopotek's spectra also show transitions to the $\Omega=2$ levels for increasing $J$, although they are much less intense than the $\Omega=1$ transitions. We searched for similar transitions approximately $40 \mathrm{~cm}^{-1}$ below those shown in Figure 2, but saw no evidence of the $\Omega=2$ state. This again shows that our molecular beam was at a very low rotational temperature.

\section{$C O \mathrm{~d}^{3} \Delta_{i}\left(\mathrm{v}^{\prime}=20, \mathrm{~J}^{\prime}=1,2,3\right)$ Radiative Lifetimes}

Radiative lifetimes are also measured for several rotational states for the $v^{\prime}=20$ level of $\operatorname{CO} d^{3} \Delta_{i}$. Figure 3 shows the results for this measurement. The log-linear plot is over a range of three Napierian decades. The PMT delay times range from $0.2 \mu$ s to $4.0 \mu$ s and a straight line fit indicative of a single exponential decay is seen. Table 2 lists the lifetimes measured. As these data show the lifetime is independent of $J$ and is: $\tau=1.70 \pm 0.08 \mu \mathrm{s}$.

Our lifetime for the $v^{\prime}=20$ level follows the trend seen at other vibrational levels, namely that the lifetime decreases as the vibrational level increases. ${ }^{31}$ Higher vibrational states generally have higher transition frequencies, resulting in a decreased radiative lifetime. We attempted to pattern the lifetime data using the reflection model. ${ }^{32}$ This method involves estimating the Frank-Condon overlap 


\section{CO Lifetimes}

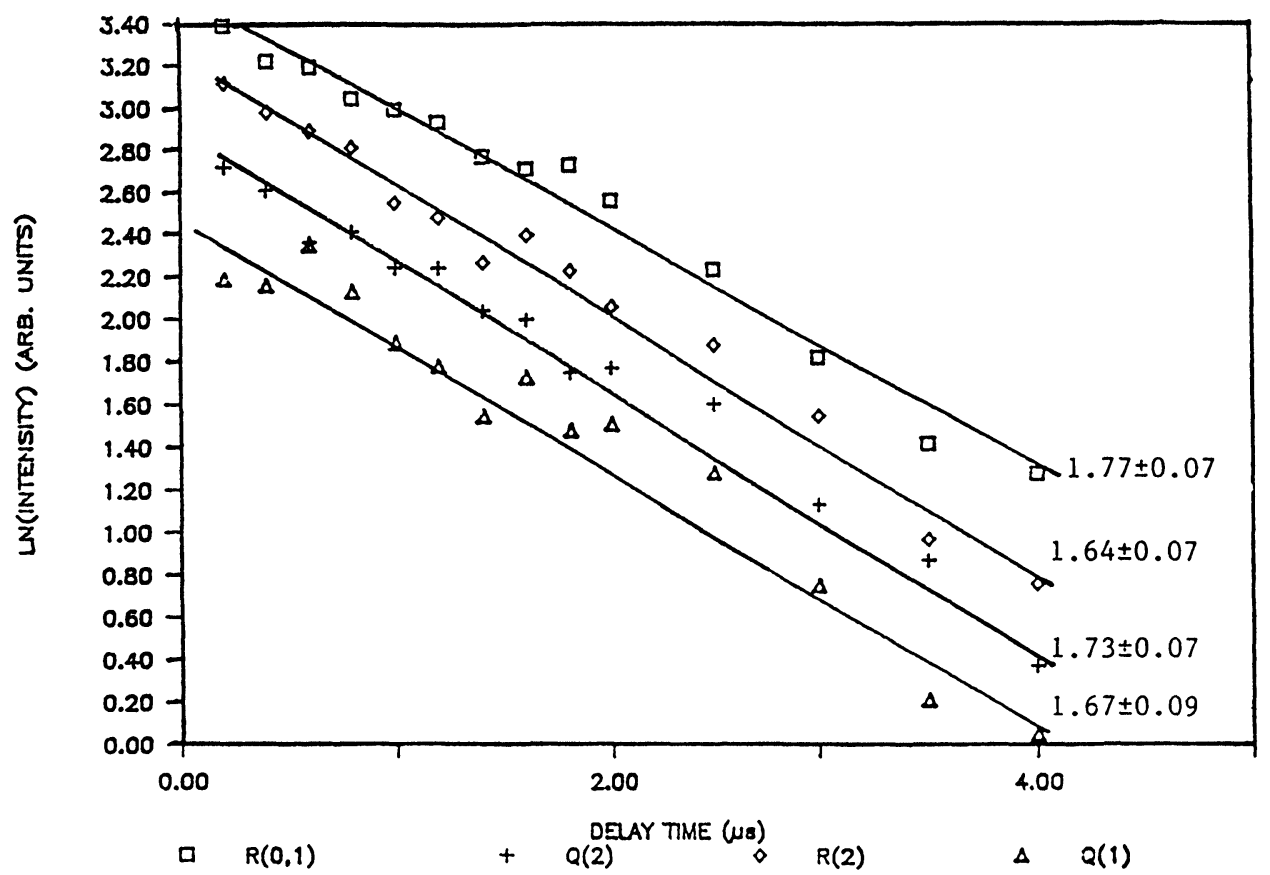

Figure 3 Radiative lifetimes for the $\operatorname{CO} d^{3} \Delta_{i}$ state. Lifetimes are the negative reciprocal of the slope of each line as determined by a linear least squares fit to the data.

Table 2 Radiative lifetimes for $\operatorname{CO} d^{3} \Delta_{i}(v=20)$

\begin{tabular}{lll}
\hline Frequency $\left(\mathrm{cm}^{-1}\right)$ & Assignment & Lifetime $(\mu \mathrm{s})$ \\
\hline 80,028 & $R(0), R(1)$ & $1.77 \pm 0.07$ \\
$80,026.5$ & $R(2)$ & $1.64 \pm 0.07$ \\
$80,023.7$ & $Q(1)$ & $1.67 \pm 0.09$ \\
$80,020.2$ & $Q(2)$ & $1.73 \pm 0.07$ \\
\hline
\end{tabular}

integral as being proportional to the average of the energy separations at the turning points of the upper vibrational level. This model is most useful for high vibrational states where the probability density function if most prominent at the turning points. The data gathered by Van Sprang et al. ${ }^{33}$ leads to predicted lifetimes greater than those observed at high vibrational levels. If the analysis were skewed to represent only high vibrational levels $(v>9)$ and forced to go to zero lifetime for infinite energy, a closer fit is achieved. Under these conditions the predicted lifetime for $v^{\prime}=20$ is $2.3 \pm 0.4 \mu \mathrm{s}$. Although this is in the neighborhood of our measured lifetime of $\tau=1.70 \pm 0.08 \mu \mathrm{s}$, there are significant differences that may be caused by perturbations as well as the lack of sophistication in the model. 
The selection rules for heterogeneous perturbations require that $\Delta \Omega=0$. Since $\Omega=1$ for the $A^{1} \pi$ state, only the ${ }^{3} \Delta_{1}$ sublevel can be involved in perturbations. Expression 2 gives a prediction for the fraction of $A^{1} \pi$ character in $d^{3} \Delta_{i}(v=20)$. Assuming that the $2.3 \mu \mathrm{s}$ lifetime given by the reflection model is valid, a $1.70 \mu \mathrm{s}$ lifetime represents a $0.15 \% A^{1} \pi$ admixture. This is only an upper limit on the $A^{1} \pi$ admixture, because $A^{1} \pi$ was assumed to be the only perturbing state. The lifetime is shorter for even larger perturbations. Slanger and Black noted a wide range of lifetimes for $v=5$ from 0.058 to $6.4 \mu$ s corresponding to $A^{1} \pi$ characteristics from $17 \%$ to $0.15 \%$. These radiative lifetimes decreased with increasing $J$. Our data show very little variation, but we are only able to make measurements on $J^{\prime}=1,2$, and 3; and low rotational quantum numbers exhibit the least variation, because the separation of rotational levels is the smallest.

\section{CONCLUSIONS}

The techniques of VUV generation by four-wave mixing and supersonic expansion are used together to find the lifetime of the $\operatorname{CO} d^{3} \Delta_{i}(v=20)$ excited state. Direct transitions to this state had never been previously reported. The four-wave mixing scheme has the following attributes: high intensity, tunability, and narrow bandwidth. These attributes allow us to acquire spectra with a bandwidth less than $1.0 \mathrm{~cm}^{-1}$ at frequencies near $80,000 \mathrm{~cm}^{-1}$. We are able to characterize a supersonic expansion by using VUV absorption from the ground state of $\mathrm{CO}$. The cooling is efficient enough to lower the rotational temperature to approximately 9 Kelvin as measured, which agrees well with the temperature predicted for a supersonic expansion.

The data indicate a rotationally independent lifetime of $1.70 \pm 0.08 \mu \mathrm{s}$. This result is consistent with Van Sprang's measured lifetimes for the vibrational states from $v=2$ to $v=16$. A comparison between the measured lifetimes for the upper vibrational states and those predicted by the reflection model shows qualitative agreement but the model typically overestimates the measured lifetimes. Possible sources of this discrepancy are the crude way the Frank-Condon overlap integrals are estimated and perturbations by nearby electronic states. We estimate an upper limited for the $A^{1} \pi$ admixture to the $0.15 \%$.

\section{References}

1. P. Helminger, F. C. DeLucia and W. Gordy, Physical Rev. Lett. 25, 1397 (1970).

2. G. Herzberg and K. N. Rav, J. Chem. Phys. 17, 1099 (1949).

3. Lova and Krupenie, J. Chem. and Phys. Ref. Data, 3, 245 (1974).

4. R. H. Tipping, J. Molec. Spectros. 61, 272 (1976).

5. K. Watanabe, M. Zelikoff and E. C. Y. Inn, Geophys. Res. Papers, (US) No. 21.

6. R. W. Field, Thesis, Harvard University, 1971.

7. T. G. Slanger and G. Black, J. Chem. Phys. 51, 4534 (1969).

8. K. N. Rav, Astrophys. J. 110, 304 (1949).

9. J. D. Simmons and S. G. Tilford, J. Res. Nat. Bur. Stand. Sect. A, 75, 455 (1971).

10. For overview see: S. G. Tilford and J. D. Simmons, J. Phys. Chem. Ref. Data, 1, 147 (1972). 
11. G. Herzberg, Molecular Spectra and Molecular Structure Vol. 1: Diatomic Molecules, (Van Nostrand Reinhold Company, New York, NY, 1950), p. 452.

12. R. T. Birge, Nature, 116, 170 (1925).

13. R. K. Asundi, Proc. Ind. Acad. Sci. A. 12, 491 (1940).

14. G. Herzberg, T. J. Hugo, S. G. Tilford and J. D. Simmons, Can. J. Phys. 48, 3004 (1970).

15. L. Gero and F. Szabo, Ann. Phys. 35, 597 (1939).

16. R. S. Mulliken, Can. J. Chem. 36, 10 (1958).

17. H. Lefebyre-Brion, C. M. Moser and R. K. Nesbet, J. Chem. Phys. 34, 1950 (1961).

18. P. K. Carroll, J. Chem. Phys. 36, 2861 (1962).

19. R. G. Fowler and T. M. Holzberlein, J. Chem. Phys. 45, 1123 (1966).

20. T. Wentink, Jr., E. P. Marram, L. Isaacson and R. J. Spindler, AFWL Tech. Rpt. 67-30, 1, Nov. 1967.

21. T. G. Slanger and G. Black, J. Chem. Phys. 58, 194 (1973).

22. W. C. Paske, J. R. Twist, A. W. Garrett and D. E. Golden, J. Chem. Phys. 72, 6134 (1980).

23. B. Girard, N. Billy, J. Vigue and J. C. Lehmann, Chem. Phys. Lett. 92, 615 (1982).

24. S. V. Filseth, R. Wallenstein and H. Zacharias, Opt. Commun. 23, 231 (1972).

25. R. Mahon and F. S. Tomkins, IEEE J. Quantum Electron. QE-18, 913 (1982).

26. Robert C. Weast, ed., Handbook of Chemistry and Physics, (CRC Press, Cleveland, OH, 1976), p. E-76.

27. S. G. Tilford and J. D. Simmons, J. of Physical and Chemical Ref. Data, 1, p. 147 (1972).

28. Ref. 11, p. 208.

29. Bruce Kay, Doctoral Thesis, University of Colorado, 1982.

30. P. Klopotek and C. R. Vidal, Can. J. Phys. 62, 1426 (1984).

31. Ref. 11, p. 20.

32. J. N. Bardsley and M. A. Biondi, Adv. At. Mol. Phys. 6, 1 (1971).

33. H. A. Van Sprang, G. R. Mohlmann and F. J. de Heer, Chem. Phys. 24, 429 (1977). 